\title{
REAL-TIME TERRAIN RENDERING WITH INCREMENTAL LOADING FOR INTERACTIVE TERRAIN MODELLING
}

\author{
Simon van den Hurk and Wallace Yuen and Burkhard C. Wünsche \\ Department of Computer Science, University of Auckland, Private Bag 92019, Auckland, New Zealand \\ \{svan071,wyue013\}@aucklanduni.ac.nz,burkhard@cs.auckland.ac.nz.
}

Keywords: $\quad$ terrain rendering, terrain modelling, sketch-based interfaces, GPU-acceleration

\begin{abstract}
Real-time terrain rendering techniques usually employ static data structures and do not allow interactive modification of the terrain. In this paper we describe a real-time geometric clipmapping terrain rendering technique for large terrains which allows incremental updates of the underlying data structure. We have combined the method with an interactive sketch-based terrain modelling technique. The clipmap data structure is updated during runtime to synchronise the terrain visualization with changes to the underlying digital elevation map. Tests and examples demonstrate the advantages of our method over traditional approaches. Disadvantages and limitations are discussed and suggestions for future work are presented.
\end{abstract}

\section{Introduction}

Terrains are an essential part of virtual environments in computer games, movies, visual impact studies, architecture, urban design and archaeology. In order to achieve real-time rendering multi-resolution representations are necessary. A popular representation is to use a regular grid of height values, also called Digital Elevation Map (DEM), where grid points are connected by triangles. A multi-resolution representation represents the same height field using different layers with a decreasing number of triangles with increasing size. When rendering the terrain regions close to the view point are represented in high resolution, and regions far away in low resolution, so that the size of triangles projected onto the view plane is approximately constant for the entire terrain.

In most applications the underlying multiresolution terrain data structures are static and do not allow modification of the terrain in real-time. In recent years an increasing number of applications have been developed requiring interactive terrain modelling, e.g. in architecture, geology, and archaeology (Keymer et al., 2009). In this paper we describe a real-time geometric clipmapping terrain rendering technique for large terrains which allows incremental updates of the underlying data structure.
Section 2 reviews existing terrain rendering techniques. Section 3 introduces the geometric clipmapping algorithm, which forms the foundation of our proposed technique. Section 4 presents our solution and section 5 presents its evaluation. We conclude this paper and suggest directions for future work in section 6 .

\section{Literature Review}

Terrain rendering techniques have been extensively studied since the mid 1990s and can be categorised into methods using tile based data structures, quad-tree based triangle hierarchies and outof-core approaches. Initial work improved computation time by reducing the level of detail with increasing viewpoint distance (Lindstrom et al., 1995; Duchaineau et al., 1997). De Boer recognised that rendering speed can be improved dramatically by using graphics hardware, which requires different data structures and algorithms. The resulting Geometrical MipMapping (de Boer, 2000), divides the terrain in multiple chunks of different levels of detail. These chunks are then updated as the user moves throughout the simulation. Additional work involving outof-core terrain rendering (Schneider and Westermann, 
2006) uses tiles and a nested mesh hierarchy to avoid mesh re-triangulation. A comprehensive survey of terrain rendering techniques is presented in (Pajarola and Gobbetti, 2007).

Early work on terrain editing includes multiresolution detail patches devised by (He et al., 2002). (Atlan and Garland, 2006) modify the terrain in realtime by specifying editing strokes, in which quadtree hierarchy is used to represent the heightmap for multiresolution editing. (Bhattacharjee et al., 2008) improve performance by utilising the GPU for rendering and editing the terrain simultaneously. The authors use a fragment shader to operate on each height value, and every time the terrain undergoes deformation or modification, the parameters of the actual process are parsed into the shader, which could be a result of simulation of terrain dynamics or direct editing performed by user using input devices such as mice.

(Dan et al., 2009) discuss various ways of terrain editing, including geometric editing and texture editing. Similar to (Bhattacharjee et al., 2008), they also modify the height point, but add natural looking variations by defining new height values using an outer and inner radius and a parameter determining the radius. We add to this work by providing an incremental update technique which enables interactive terrain modelling by minimizing data transfer and data structure updates in GPU memory.

\section{Geometry Clipmaps}

Geometry Clipmaps is a level-of-detail GPUbased terrain rendering technique (Losasso and Hoppe, 2004; Asirvatham and Hoppe, 2005). It uses multiple representations of the same terrain at different resolutions to increase the efficiency of the rendering process. The clipmaps data structure is broken into several layers, each of which contains a higher resolution than the layer below it. These layers are arranged by centering them about the viewpoint and then rendering them. The terrain data is stored in a vertex buffer object and updated as the viewpoint moves throughout the simulation. This grid of data is stored using an offset into the vertex buffer object to allow for a toroidal access. By using a toroidal index the vertex buffer object is able to only update a single column or row rather than moving all rows or columns within the vertex buffer object to a new location.

The update process examines each level in the clipmap, starting from the lowest resolution and iterating to the highest. Each level uses the viewpoint of the camera to determine an active region of the clipmap. If this active region differs from the previ- ous update's active region, then the vertex buffer object is updated, and the data will be synchronised with the terrain data that is stored within the RAM memory. When the terrain is rendered, each clipmap layer renders a ring section within which the next clipmap layer is rendered. The resolution decreases with increasing distance from the viewpoint.

The most important aspect of the clipmaps data structure is the way clipmap layers are blended to create smooth transitions between them. The vertex information of the terrain stored by each clipmap layer includes not only the $\mathrm{x}, \mathrm{y}$, and $\mathrm{z}$ coordinates of each point in the terrain, but also an additional channel containing the height of the parent layer at this point. During the render method, an alpha value is calculated to interpolate between the vertex height and the parent vertex height. The alpha value approaches one for the vertices that are closer to edge of the clipmap, therefore aligning the edge of the clipmap with the edge of its parent clipmap layer.

\section{Design}

In order to interactively model terrains we need a technique with suitable data structures, which can be modified to support interactive updates, and with as few constraints as possible when using it. After careful analysis we chose the Geometry Clipmaps algorithm (Asirvatham and Hoppe, 2005). A major advantage is that the algorithm performs well when viewed from a top down perspective, which is important during modelling, e.g., to add and view terrain structures such as mountain ranges and rivers. The algorithm also loads large sections of terrain, allowing for a fast rotation of the viewpoint without a large computational requirement. The algorithm merges well with the sampling technique that the sketch based input provides. The geometry clipmaps algorithm uses multiple levels of detail and the sketch based input can provide these different levels by sampling the contours at different resolutions. Real-time incremental updating of the terrain is achieved by computing small clipmap sections fitting into multiple resolution representations and as such minimizing the amount of data passed between the RAM and graphics card memory.

\subsection{Data Structures}

The data for the surface of the terrain will be stored within the RAM memory during the runtime of the program. In order to access the terrain data and provide it to the clipmaps data structure, we wrote the Terrainsurface interface. 
The clipmaps algorithm requires different representations of the same terrain at different resolutions. The desired representations are obtained by calling the methods within this interface with a Dimension parameter. The terrain data is stored in RAM using a one-dimensional array of floats. This packed data is then accessed using offsets within the data. A data stride value is required to determine the byte offset between vertex and normal data. The interface describes the methods providing access to the different types of data required by the clipmaps algorithm:

- Vertex information.

- Normal Information.

- Parent Normal Information.

- Offsets to the position of the above information within the single dimensional array.

By using offsets into the one dimensional array the interface provides flexibility for implementations which already contain a set structure for the order of the data stored in the packed array.

The implementation of the clipmaps algorithm is split into two separate classes: Clipmaps and Clipmap (van den Hurk et al., 2011). The Clipmaps class provides the interaction with the clipmaps concept and is the class instantiated by the end user. This class manages the updating of all levels of the clipmap data structure, as well as providing simple method calls to render the entire data structure.

The Clipmap class provides implementation for a single layer of the clipmaps data structure. Each layer of the clipmap is stored in a series of vertex buffer objects within the memory of the graphics card. This class provides core methods to create these vertex buffer objects, and to update them as the viewpoint is moved throughout the scene. It also provides methods to render the terrain and perform the frustum culling to improve the efficiency.

For this specific project there are some notable changes from the original geometry clipmaps implementation. The most important is that no compression is done upon the terrain data stored in RAM memory, since the compression algorithm used within the original paper is quite quite slow and would prevent interactive frame rates. Another modification is that the lowest level of the clipmap data structure has been changed to always render, regardless of the camera position. In the original implementation the entirety of the terrain is only rendered when the viewpoint is in the centre of the terrain. By enforcing the lowest level to always be drawn, the entire terrain is therefore always visible at the lowest resolution regardless of the viewpoint. Lastly, this implementation allows non-square shaped clipmaps to be defined, so that the size of the clipmaps can be rectangular such as $256 \mathrm{x}$ 512.

\subsection{Incremental Updates}

An interactive terrain modelling system must show any modifications to the terrain in real-time. The visual feedback will assist the users with editing the terrain. In order to achieve this, only small rectangular sections enclosing the modified regions are updated.

\subsubsection{Clipmaps Section Update}

Updates of the terrain data will change the information stored in RAM memory that is interacted with through the Terrainsurface interface. After a section of the terrain stored in RAM memory is updated, the corresponding data within the graphics card memory must also be updated. This functionality is provided through an UpdateSection method within the Clipmaps class. This method works by defining a rectangular region that is to be updated and a Dimension variable which specifies at which resolution the rectangular region is specified.

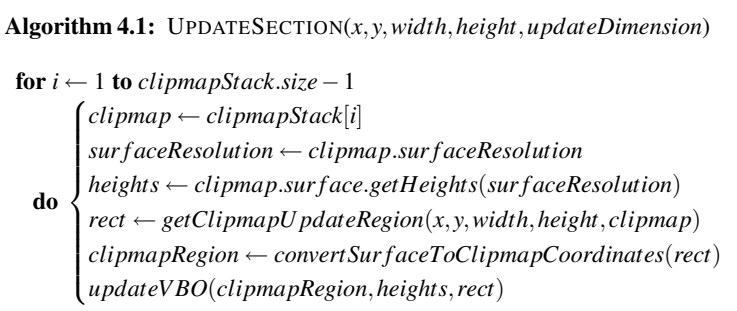

As shown in algorithm 4.1, the method iterates through all clipmap layers in the clipmap stack and determines the appropriate rectangular region to be updated by scaling the co-ordinates for each level depending on the surface resolution. The method then performs a standard update for this region, and lastly synchronises the data on the graphics card with the newly updated terrain data in the RAM memory. By using this method, the amount of data required to be transferred between the RAM memory and the graphics card memory is reduced to a minimum, reducing rendering time and saving bandwidth for other applications.

\subsection{Shaders}

The clipmaps implementation requires specific shaders to correctly render the desired terrain. These shaders were written in the OpenGL Shading Language (GLSL). 


\subsubsection{Vertex Shader}

The vertex shader takes two additional parameters viewCoord and activeRegionSize, which are the same for every rendered vertex. These parameters are both of type vec 2 and declared uniform as they do not change between each rendered vertex. viewCoord specifies the position of the camera and is used to determine around which point the alpha blending is centered. The other parameter activeRegionsize specifies the width and height of the active region which is to be drawn. Combining these two variables, the shader is able to calculate the alpha value required to blend between the height value of this clipmap and its parent. This is done using the formula specified by (Losasso and Hoppe, 2004):

$$
\alpha_{x}=\min \left(\max \left(\frac{\left|x-v_{x}^{l}\right|-\left(\frac{x_{\max }-x_{\min }}{2}-w-1\right)}{w}, 0\right), 1\right)
$$

This formula calculates the difference between the vertex position $v$ and the position of the camera within this layer $v_{x}^{l}$, and then subtracts half of the region size and the blend width $w$. A blend width of 10 was found to produce suitable results, as lower blend width values tend to produce less smooth results between two clipmaps, and higher blend width values require unnecessarily more calculations. This calculated value is then divided by the blend width $w$ and finally the result is clamped to be within a range of zero to one. A similar formula is also used for $\alpha_{y}$ and the final alpha value is calculated as the maximum of $\alpha_{x}$ and $\alpha_{y}$. Using this calculated alpha value the blended height can now be determined. This is done using a linear interpolation between the height value of the current clipmap and the height value of the parent clipmap stored in the fourth channel of the position vertex gl_Position. The formula to calculate the final height value is:

$$
\text { blendedHeight }=(1-\alpha) * h e i g h t^{l}+\alpha * h e i g h t^{l+1}
$$

To avoid slight rounding errors with the final blended height the alpha value was rounded up to 1 if the value was close to that number. This ensures the border vertices are completely rendered using the parent height and as such provide a seamless integration with the surrounding clipmap level.

The shaders also calculate the lighting and final colour of the pixel, which requires blending normals so that they correspond to the blended height values. Normal blending uses the $\alpha$ value for blending heights in order to linearly interpolate between the normal of this layer and the normal of the parent layer:
blendedNormal $=(1-\alpha) *$ normal $^{l}+\alpha *$ normal $^{l+1}$

This blended normal must then be normalized in order to ensure the lighting is correctly calculated.

\subsubsection{Fragment Shader}

The fragment shader is used to calculate the final colour of the pixel fragment that is to be drawn to the screen. The intensity of the fragment must be calculated and then merged with the colour value from the texture. When calculating the intensity the normal must be normalized once again. This is because this normal value is a linear interpolation between two of the normals provided with vertices. The linear interpolation does not guarantee a normalized vector and so this normalization must be performed manually. Combining these two values produces the final colour that is to be rendered to the screen.

\subsection{Rendering Optimisations}

\subsubsection{Active Regions}

The active region defines the section of the clipmap level that is to be rendered to the screen. The dimensions of this active region must lie within the clipmap. During the update method of the clipmap, the active region is recalculated if the position of the viewpoint has moved. To ensure that the rendering of the clipmap aligns with the parent layer, the active region must be enlarged so that its vertices are shared by the current and the parent layer. The clipmap layer which defines the finest resolution is drawn as a single rectangular block using triangle strips. All other layers are a ring shape, with a hole in the centre where the next clipmap layer is drawn. We use eight blocks rather than four as in (Losasso and Hoppe, 2004), which reduces computations during frustum culling.

\subsubsection{Frustum Culling}

The frustum culling process reduces the amount of computation required in the rendering process. This is done by determining which sections of the clipmap need to be drawn given the current orientation and position of the viewpoint. Two approaches were tried in order to perform this frustum culling.

The first was to project the points of each of the eight segments in the clipmap onto a horizontal $\mathrm{x}-\mathrm{Z}$ plane. Then the points of the view frustum were also projected onto this plane. Following this, the points of each region were tested for containment within the oriented bounding box of the axis projected frustum. 
If at least one of the points was contained, then the region was considered necessary for rendering. This method proved to be the least successful due to the nature of the oriented bounding box. The calculated bounding box would often include many points within the region, which were not required for the rendering process. This over inclusion of points displaced any advantage that might have been gained by using the efficient containment detection provided by the bounding box.

The second implemented technique for frustum culling approximated the viewing frustum using six planes. The bounds of each region segment were then tested for containment within these six planes. This proved to be efficient as it only requires a simple distance calculation to determine upon which side the plane a point lies. Furthermore this method determines containment within the frustum far more accurately than using bounding boxes. If any of the segment points was found to be within the view frustum, then that segment of the clipmap is rendered.

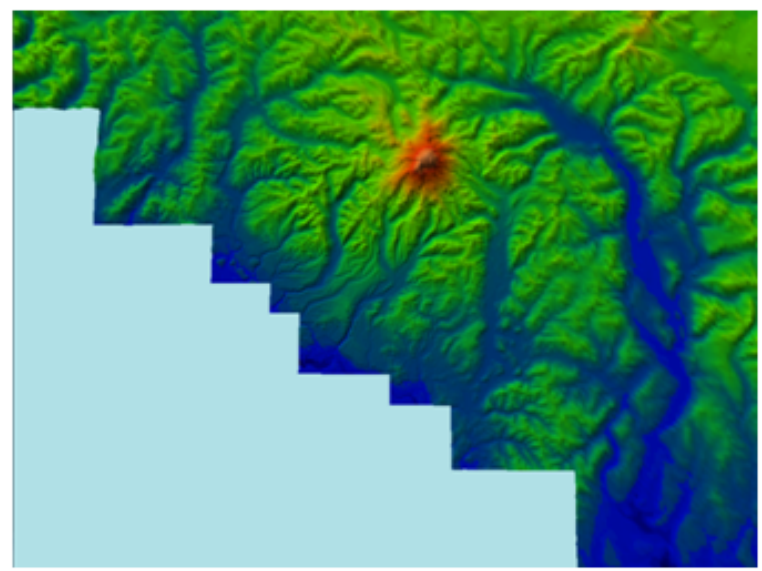

Figure 1: An aerial view of the clipmaps algorithm being run with the culled segments removed from the rendering process.

\subsubsection{Viewpoint-Based Culling for Interactive Modelling}

In the original geometry clipmaps algorithm the high resolution clipmap levels surround the camera position. This allows a user to see high levels of detail in the terrain in their immediate vicinity. It also guarantees a high rendering speed should the user rotate rapidly or wish to quickly look in the opposite direction. The original motivation for this design comes from flight simulators where the user frequently looks out of varying viewports of the cockpit. For interactive terrain modelling we use a top-down view which rotates around a point of interest.

The frustum culling that is performed by the clipmap levels to determine which segments should be rendered requires still the original position of the camera. Using the point upon which the camera rotates would not provide a correct rendering output.

It is important to note that this modification contains a flaw. Should the camera be sufficiently far away from the central rotation position and then tilt to a low angle such that the camera skims across the terrain with its viewpoint then the high resolution clipmaps will lie far away from the camera position and the low resolution terrain will be visible to the user. A possible method to avoid this situation would be to interpolate between the point of rotation and the camera position depending on the angle between the camera and the rotate position and the plane that the terrain resides upon.

\section{Results}

The performance of the incremental updates were measured by using multiple resolutions with varying clipmap size. These tests were run with a Nvidia GeForce GT 330M 512MByte graphics card. Each test changed the height of a $20 \times 20$ area of pixels in $60 \mathrm{fps}$ using the glutIdleFunction to control the frequency. We found that the frame rate can be improved by up to $45 \%$ using incremental updates. We have used the rendering algorithm in combination with a technique for the sketch-based modelling of rivers and lakes and achieved an interactive performance and pleasing results as illustrated in figure 2 . More detailed results can be found in (van den Hurk et al., 2011).

As indicated by the pictures the algorithm is well suited for sketch-based input. Apart from improved performance it also removes the restriction of having fixed sized clipmaps, thus allowing the shape of the clipmaps to be rectangular, and allowing the difference in resolution between clipmaps to be a multiple of two, rather than exactly double. A current disadvantage that may arise is that for very large update regions the terrain in the RAM might be only partially updated and not synchronised with the graphics card. Also, due to compiler restrictions for the size of one-dimensional arrays, the maximum terrain size is currently 53687091 data points, which is equivalent to a clipmap level size of $7327 \times 7327$ vertices. Larger terrains could be represented by multiple instances of the Clipmaps class in combination with an out-of-core terrain rendering technique. 

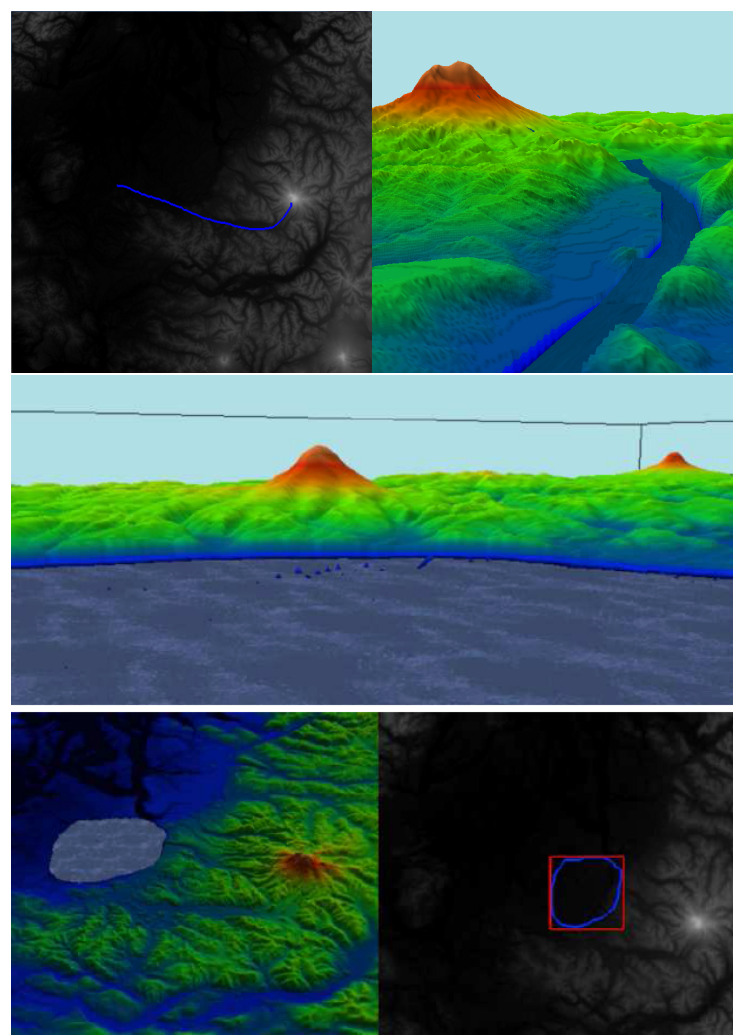

Figure 2: A river (top) and lake (center and bottom) added to a high-resolution terrain using $2 \mathrm{D}$ sketch input.

\section{Conclusion and Future Work}

An implementation of the geometry clipmaps data structure has been developed, which allows incremental updates to the terrain at run-time in order to enable interactive editing of large terrains. The update regions can be of arbitrary size making the technique suitable for applications requiring constant small changes, such as sketching a river, and for large changes, such as inserting a new mountain range. We extended the underlying Geometry Clipmap approach to allow arbitrary view points, including a birds-eye perspective, which is useful for terrain editing.

Our results demonstrate that terrains can be edited and rendered at interactive frame rates in high resolutions. The main limitation is the maximum allowed terrain size due to compiler restrictions and GPU memory limitations. This could be overcome by employing concepts from out-of-core terrain rendering techniques. Additional future work includes the use of 2D textures, including multi-texturing in order to combine large-scale texture variations with terrain details.

\section{REFERENCES}

Asirvatham, A. and Hoppe, H. (2005). Terrain rendering using GPU-based geometry clipmaps. GPU Gems, 2:27-46.

Atlan, S. and Garland, M. (2006). Interactive multiresolution editing and display of large terrains. Computer Graphics Forum, 25(2):211-223.

Bhattacharjee, S., Patidar, S., and Narayanan, P. (2008). Real-Time Rendering and Manipulation of Large Terrains. In Computer Vision, Graphics \& Image Process, 2008. ICVGIP'08, pages 551-559.

Dan, L., Yingsong, H., M., D., and Xun, L. (2009). The Research and Implementation of Interactive Terrain Editing and Crack Elimination. In Proc. of Computational Intelligence and Software Engineering (CiSE 2009), pages $1-4$.

de Boer, W. (2000). Fast terrain rendering using geometrical mipmapping. http://www.flipcode.com/ articles/articlegeomipmaps.pdf.

Duchaineau, M., Wolinsky, M., Sigeti, D., Miller, M., Aldrich, C., and Mineev-Weinstein, M. (1997). ROAMing terrain: real-time optimally adapting meshes. In Proceedings of Visualization '97, page 88.

He, Y., Cremer, J., and Papelis, Y. E. (2002). Real-time extendible-resolution display of on-line dynamic terrain. In Proc. of Graphics Interface, pages 151-160.

Keymer, D., Wuensche, B., and Amor, R. (2009). Virtual Reality User Interfaces for the Effective Exploration and Presentation of Archaeological Sites. In Proc. of CONVR, pages 139-148.

Lindstrom, P., Koller, D., Hodges, L., Ribarsky, W., Faust, N., and Turner, G. (1995). Level-of-detail management for real-time rendering of phototextured terrain. Graphics, Visualization \& Usability Center, Georgia Institute of Technology, Technical Report GITGVU95-06.

Losasso, F. and Hoppe, H. (2004). Geometry clipmaps: terrain rendering using nested regular grids. In Proc. of SIGGRAPH 2004, pages 769-776.

Pajarola, R. and Gobbetti, E. (2007). Survey of semi-regular multiresolution models for interactive terrain rendering. The Visual Computer, 23(8):583-605.

Schneider, J. and Westermann, R. (2006). GPU-friendly high-quality terrain rendering. Journal of WSCG, 14(1-3):49-56.

van den Hurk, S., Yuen, W., and Wünsche, B. C. (2011). Real-time terrain rendering with incremental loading for interactive terrain modelling. Graphics group technical report \#2011-003, Department of Computer Science, University of Auckland. http: //www.cs.auckland.ac.nz/ burkhard/Reports/ GraphicsGroupTechnicalReport2011_003.pdf. 